\title{
Satisfaction with Delivery Services Offered under the Free Maternal Healthcare Policy in Kenyan Public Health Facilities
}

\author{
C. M. Gitobu (D), P. B. Gichangi, and W. O. Mwanda \\ University of Nairobi, Kenya \\ Correspondence should be addressed to C. M. Gitobu; cosmgambi@gmail.com
}

Received 31 October 2017; Revised 28 February 2018; Accepted 17 April 2018; Published 22 May 2018

Academic Editor: Evelyn O. Talbott

Copyright ( 2018 C. M. Gitobu et al. This is an open access article distributed under the Creative Commons Attribution License, which permits unrestricted use, distribution, and reproduction in any medium, provided the original work is properly cited.

\begin{abstract}
Background. Patients' satisfaction is an individual's positive assessment regarding a distinct dimension of healthcare and the perception about the quality of services offered in that health facility. Patients who are not satisfied with healthcare services in a certain health facility will bypass the facility and are unlikely to seek treatment in that facility. Objective. To determine satisfaction level of mothers with the free maternal services in selected Kenyan public health facilities after the implementation of the free maternal healthcare policy. Methods. Data was collected through a quantitative exit survey questionnaire. The respondents were mothers who had delivered in the health facilities and were waiting to leave the health facilities after discharge. The sample included 2,216 mothers in 77 public health facilities across 14 counties in Kenya under tier 3 and tier 4 categories. The number of respondents to be interviewed was proportionately arrived at based on each health facility's bed capacity. Results. The study established a satisfaction rate of $54.5 \%$ among the beneficiaries of the free maternal healthcare services in the country. Mothers benefiting from the free delivery services were satisfied with communication by the healthcare workers, staff availability in the delivery rooms, availability of staff in the wards, and drug and supplies availability $(>56 \%)$ but unsatisfied with consultation time, cleanliness, and privacy in the wards $(<56 \%)$. High education levels and lengthy stay in healthcare facilities were negatively associated with the satisfaction with the free delivery services $(P<0.05)$. Conclusion. There is a high satisfaction with the free maternal healthcare services in Kenya. However, the implementation of the free maternal healthcare policy was associated with low privacy, poor hygiene, and low consultation time in the health facilities. Therefore there is need to address these service gaps so as to attract more mothers to deliver in public health facilities.
\end{abstract}

\section{Background}

Millennium Development Goal (MDG) 5-Target 5A called for the reduction of maternal mortality ratio by threequarters between 1990 and 2015; however, to date, reduction of avoidable pregnancy related mortalities still remains one of the greatest health challenges and priorities globally [1]. In the year 2015, there were 303,000 maternal mortalities worldwide [1]. Despite numerous global efforts to curb these pregnancy related deaths, they remain disturbingly high in developing countries yet they are almost always preventable through the attendance of pregnancy and deliveries by skilled healthcare professionals in adequately supplied and equipped health facilities [2].

Maternal mortality has been shown to occur as a result of direct obstetric causes (73\%) and indirect causes (27\%) with the major causes being haemorrhage (27.1\%), hypertensive disorders (14\%), sepsis (10.7\%), abortion (7.9\%), and embolism (3.2\%) with these figures showing regional variations [3]. Thus, the Global Strategy for Women's, Children's and Adolescents' Health (2016-2030) envisions a world in which every woman, child, and adolescent in every setting realizes his/her rights to physical and mental health and wellbeing, has social and economic opportunities, and is able to participate fully in shaping prosperous and sustainable societies [4].

Kenya is still struggling to reduce maternal mortality rate in the country which stands at 362 deaths for every 100,000 live births [5]. This high maternal mortality rate has been partially attributed to unskilled deliveries in the country (38\%) which are conducted outside health facilities [5]. Low utilization of maternal healthcare services in Sub-Saharan Africa 
has for long been linked to the high cost of the services $[6,7]$. In Kenya, cost of maternal and child health services has also been cited as one of the obstacles to service utilization $[8,9]$.

Unaffordable health services globally are a result of inadequate health financing care models which often result in catastrophic expenditures throughout pocket payments for health services [10-16]. At the 13th of December 2017, at least half of the world's population could not access essential health services due to cost implications while 800 million people globally spend at least 10 percent of their household budgets on health expenses for family members [17]. For this reason, universal health coverage has been suggested as an additional measure to promote access to health services by all individuals and communities without suffering financial hardships and it entails the full spectrum of essential, quality health services, from health promotion to prevention, treatment, rehabilitation, and palliative care $[18,19]$.

Economic related reasons are a significant barrier to delivering at health facilities in many regions in Kenya $[15,16]$. Previous assessments in Kenya have established that up to $11 \%$ of women do not deliver in health facilities due to the cost of skilled delivery services [20]. Catastrophic spending on health is approximated to be $6.2 \%$ in Kenya given that only $17.1 \%$ of Kenyan households had medical insurance covers in 2013 [21] and 20.6\% had medical insurance covers in 2014 [22]. The Kenya household expenditure survey 2013 reported that wealthy individuals in the richest wealth quintile were more likely to seek heathcare services when compared to poorer individuals in low wealth quintiles [21]. This report further shows that the richest wealth quintile were more likely to utilize inpatient care specifically, 56 admissions per 1,000 population against 28 admissions per 1,000 population for the poorest quartile.

Based on the observations of economic barriers to health services utilization observations, there have been proposals and resolutions to streamline health financing systems to promote access to quality health services [23]. In addition, the 2006 healthcare financing strategy for the African countries by WHO emphasizes the expanded coverage of healthcare services to ensure accessibility by all African populations [24]. As a result of this, African countries such as Mali, Ghana, Uganda, South Africa, Niger, Senegal, and Burkina Faso have been undertaking healthcare financing reforms such as abolishment of user fees in health to improve the accessibility of healthcare services [25].

It is against this backdrop that the Kenyan government introduced a free maternal healthcare policy through a presidential directive on 1st June, 2013, so as to promote skilled delivery and reduce pregnancy related mortalities [26]. Through this policy intervention, mothers are supposed to deliver free of charge in Kenyan public health facilities. Public health facilities are reimbursed between 25 and 175 US dollars for every delivery whether through spontaneous vaginal delivery or caesarian section. The amount of money reimbursed to the health facilities for every delivery is based on the facility status and health systems capacity to handle complications. As such, tier 1 health facilities, where no caesarian sections, blood transfusion, or inward referral of complicated cases are carried out, receive the minimum amount for every delivery (25 US dollars). Tier 4 health facilities handle most complications from deliveries and therefore they receive the maximum 175 US dollars. It is expected that mothers will be encouraged to deliver in health facilities, maintain a good relationship with the health facilities, and continue to seek other services such as postnatal care services thus ultimately improving pregnancy outcomes. Initial assessments reveal that the free maternal health policy implementation in Kenya is faced with numerous challenges among them shortage of drugs and supplies, insufficient funding, shortage of skilled healthcare workers, noninvolvement of stakeholders in maternal health, late reimbursement of the costs incurred in providing free maternal healthcare services, heavy workloads, and demotivation of health workers $[27,28]$.

The existence of free delivery healthcare service neither promises their utilization by women nor does it guarantee optimal pregnancy outcomes or satisfaction with the services [29]. Reproductive health services should be satisfactory to mothers since they may have immediate and long-term effects on her health, subsequent utilization of delivery services, and recommendation of the services to her peers [3032]. It is for this reason that the World Health Organization (WHO) recommends a close monitoring of women's satisfaction with delivery services as a quality check to improve skilled delivery outcomes [33]. Patients' satisfaction with healthcare services is one of the measures for quality of care that has been shown to influence confidence in a health facility and the subsequent utilization of services from the facility $[34,35]$. Patients' satisfaction with quality of healthcare is the degree to which the patients' desired expectations, goals, and preferences are provided by the healthcare service providers [36]. Patients' satisfaction and dissatisfaction with healthcare services indicate their perception about the strengths and weaknesses in the service delivery [37].

Satisfaction with healthcare services in Kenya has for long been reported as low and cost of services has been given as one of the reasons for dissatisfaction with the services [32, 38-40]. Similarly, incidences of unfriendly healthcare workers as well as poor treatment of pregnant mothers in public health facilities in Kenya have been demonstrated to be a barrier in maternal healthcare seeking behaviour $[27,28,41,42]$. This study was intended to establish the satisfaction of beneficiaries with free delivery services in Kenyan public health facilities and identify factors associated with the satisfaction. Recall questions were used to establish the satisfaction with delivery services by mothers who had their previous deliveries in the public health facilities prior to the policy intervention.

\section{Methods}

2.1. Study Design. This study employed a cross-sectional analytical approach. A structured questionnaire was used to interview mothers through exit interviews in the public health facilities between July and December 2015.

2.2. Dependent Measures. Satisfaction in this study was based on the following variables: consultation time, communication, and attitude by the healthcare workers, staff availability 
in the delivery rooms, availability of staff in the wards, cleanliness in the health facilities, drug and supplies availability, and privacy in the wards. These variables were selected based on previous studies in the country which revealed that they were the major aspects of delivery services that influenced user satisfaction $[14,38,40]$.

Beneficiaries were asked to score each satisfaction element on a Likert scale of 1 to 5 (where 1 was very low, 2 was low, 3 was moderate, 4 was high, and 5 very high). The threshold for satisfaction was set as $56 \%$ of the respondents for each dependent measure given that a previous study on satisfaction in the county had established delivery service user satisfaction to be $44 \%$ with $12 \%$ of the respondents being unsatisfied with the cost of services charged [32].

2.3. Study Population. The respondents were mothers who had delivered in the health facilities, had been discharged from the health facilities, and were waiting go home.

2.4. Study Setting. Kenya is administratively divided into 47 counties [27]. The public health facilities in the country are categorized into four tiers; tier 1 is community health centres, tier 2 is primary care level health facilities, tier 3 is county and subcounty health facilities, and tier 4 is the national referral health facilities [43].

2.5. Selection of Health Facilities. Stratified multistage sampling was used to select 77 public health facilities in 14 of the 47 counties in Kenya ( 5 high risk, 5 medium risk, and 4 low risk maternal mortality risk counties based on annual maternal mortality figures for the years 2008, 2009, and 2010).

Fourteen of the forty-seven counties in the Republic of Kenya were selected for inclusion in the study after single-stage cluster sampling and subsequent simple random sampling procedures were applied [44].

The 47 counties were classified into high risk, medium risk, and low risk maternal mortality categories based on their perennial maternal mortality ratios. Of these counties, five with a high risk (maternal death to females population ratio above 0.00018 ), five with a medium risk (maternal death to females population ratio between 0.00012 and 0.000183 ), and four with a low of risk maternal mortality (maternal death to females population ratio below 0.00012) were included in the study; these studies were selected via simple random sampling. As at 2015, Kenya had a total of 234 health facilities in tiers 3 and 4 of which 97 were in the 14 selected counties, from which 77 (76 in tier 3 and 1 in tier 4) were selected (based on 5\% margin error, 95\% confidence interval and a postulated increase in satisfaction from $54 \%$ to $56 \%$ following elimination of delivery charges) through stratified multistage sampling with the maternal mortality risk, counties, status of health facilities and location being the strata (whether urban or rural as classified in the master health facility list), and the proportion of health facilities in each county $[45,46]$.

2.6. Selection of Respondents. 2,217 respondents were proportionately divided among the health facilities based on the bed capacity in each health facility (based on 5\% margin error, $95 \%$ confidence interval and a postulated $56 \%$ satisfaction rate). Systematic random sampling of respondents was carried out with the $n$th respondent in every health facility being the number of free maternal healthcare services beneficiaries eligible for inclusion into the study divided by the sample size allocated for the facility. Where a health facility had more than one maternity ward, the total population of respondents was sum number of eligible respondents in all the maternity wards.

2.7. Exclusion Criteria. Mothers who declined to participate were excluded from the study and so were mothers who had not delivered and those who had not been discharged at the time of the interviews. The respondents for the exit survey were mothers who had delivered in the selected public health facilities, were discharged, and were waiting to go home (after utilizing the free maternal healthcare services); only those who consented to participate in the exit survey were included in the study.

2.8. Recruitment Strategy. Nurses in charge of maternity wards were used to identify and recruit respondents. All the background information regarding the study (including benefits and risks) was provided to each mother; they were requested to give consent for participation in the study.

2.9. Instruments of Data Collection. The data collection tool used in this study was an interviewer who administered questionnaire for the beneficiaries of the free skilled delivery services. The questionnaire ranked the responses to satisfaction questions through a Likert scale.

2.10. Data Management and Statistical Analysis. Four research assistants collected the data after rigorous training on data collection and research ethics by the main author using the National Institutes of Health (NIH) research ethics guidelines [47]. Pretesting of the data collection tools and pilot testing of the data collection procedures were done at a level 4 health facility outside the sampling frame. Data analysis was carried out using the Statistical Package for Social Sciences (SPSS) version 23 and graphics were generated using Microsoft Excel (version 2013) while the degree of association between responses was tested at 95\% confidence interval through Pearson's Chi-square test. Data was collected in Kiswahili and English languages in the months of June to October 2015.

2.11. Ethical Considerations. Ethical approval was obtained from Kenyatta National Hospital and University of Nairobi Ethical Committee while administrative approval was obtained from the Ministry of Health headquarters in Kenya, county health officials, and the health facility administrators. Those who consented to participate in the study were interviewed in isolated rooms to ensure privacy. The study participation was voluntary; the nursing officer in every ward acted as witness to ensure that only those who gave consent were included in the study. The participants signed a consent form upon satisfactorily understanding the scope of the study and consented to participate in the study; where mothers below 18 years were encountered, they gave assent and their 


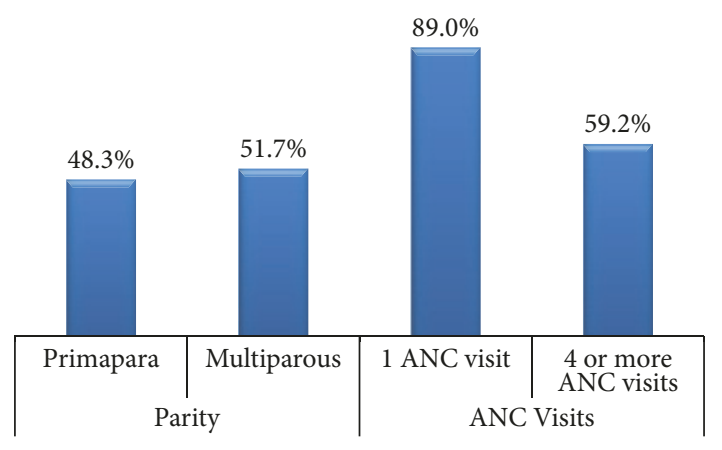

FIgURE 1: Clinical characteristics of respondents.

parents signed the consent forms. There was no direct or indirect compensation for participation in the study.

\section{Results}

3.1. Response Rate. Out of the 2,217 questionnaires administered, 2,216 were completed translating to a $99.6 \%$ response rate. Of these, $82.2 \%$ of the respondents were interviewed in tier 3 health facilities while $17.8 \%$ were interviewed in tier 4 health facility. In addition, $57.7 \%$ of the health facilities were located in urban areas while $42.3 \%$ of the health facilities were located in the rural areas.

3.2. Demographic Information of the Respondents. The study population had a median age of 26 years, a minimum age of 13 years, and a maximum age was 49 years (Table 1). Among these respondents, $72.7 \%$ were married females, $24.1 \%$ single females, $2 \%$ divorced, and $1.2 \%$ widowed. In addition, $89 \%$ of the respondents were Christians while $9.7 \%$ were Muslims, $0.5 \%$ Hindus, $0.4 \%$ subscribed to traditional believes, and $0.4 \%$ belonged to other unspecified religions. In terms of level of education levels, $27.2 \%$ of the mothers had attained a tertiary level of education, $24.3 \%$ of the mothers had completed secondary school education, and $3.2 \%$ of the respondents had never been to school.

3.3. Satisfaction with Free Maternal Healthcare Services. On analysis of previous delivery histories, $48.3 \%$ were primipara (first time mothers) while $51.7 \%$ were multiparous respondents. At least 1 antenatal clinic (ANC) visit was reported by $89.0 \%$ of the mothers. In addition, $59.2 \%$ of the mothers had made 4 or more ANC visits while $40.8 \%$ had made less than 3 ANC visits (Figure 1). Respondents got to know of the free delivery services through the media (37\%), healthcare workers $(15.7 \%)$, relatives $(22.4 \%)$, mobile outreaches $(6.9 \%)$ through neighbours $(10.7 \%)$, friends, workmates and community health volunteers (6.2\%), and undisclosed sources $(0.5 \%)$ while $0.4 \%$ were not aware of the existence of free delivery services.

The median duration of stay in the health facilities before discharge was 3 days ( 3 days for those who had spontaneous vaginal deliveries and 4 days for those who delivered via caesarian sections) while the maximum and minimum days spent in the health facilities before discharge were 70 days ( 28 days for those who had spontaneous vaginal deliveries and
70 days for those who delivered via caesarian sections) and 2 days (1 day for those who had spontaneous vaginal deliveries and 2 days for those who delivered via caesarian sections).

The study established that $54.5 \%$ of the respondents were satisfied with delivery services offered through the free maternal health policy while $30.2 \%$ and $15.3 \%$ remained neutral and were unsatisfied about the services, respectively. The only service elements that the mothers were not satisfied with $(<56 \%)$ were consultation time, cleanliness, and privacy in the wards (Table 2).

The satisfaction levels were highest in tier 4 health facility (65.1\%) when compared to tier 3 health facilities. Satisfaction with privacy was higher in tier 4 health facility $(47.6 \%)$ when compared to tier 4 heath facilities $(30.8 \%)$. There were no differences in satisfaction levels between mothers receiving free maternal healthcare services in rural and urban areas (Table 3).

Sharing of beds by mothers during their stay in health facilities was reported by $43 \%$ of the mothers while $26.8 \%$ of the mothers reported that their babies shared incubators with other babies in the health facilities. Sharing of beds and incubators was highest in tier 4 health facility at $62 \%$ and for incubators at $51.1 \%$, respectively. A large proportion of the respondents $(85.7 \%)$ reported to have received explanations for procedures carried out on them and information on drugs that were administered or prescribed (85\%) with the highest proportion being in tier 4 health facility (94.9\% and $93.2 \%$ ). In addition, $49.3 \%$ of the mothers reported to have been provided with a mosquito net while $52.8 \%$ were provided with warm water for showering.

A bivariate analysis of factors associated with satisfaction revealed that that level of facility, days spent at the health facility, number of ANC visits, time taken to access a health facility, sharing of beds, sharing of incubators, effectiveness of pain control remedies, explanation of procedures to be carried out, provision of information on drugs, quality of meals, cleanliness of sanitation facilities, provision of warm water, and waiting time before being attended to upon arrival at the health facilities were predictors of satisfaction $(P<$ $0.05)$. From these factors, a lengthy stay in the health facilities $(P=0.01)$ and high levels of education level $(P=0.01)$ were associated with dissatisfaction with the free maternal healthcare services (Table 4).

\section{Discussion}

This study was aimed at establishing users' satisfaction of delivery services offered under the free maternal healthcare policy in Kenyan public health facilities. The minimum length of stay in the health facilities was 2 days with a mean of 3 days and maximum of 70 days spent in the health facilities. This observation is in agreement with findings that $87 \%$ of free maternal healthcare beneficiaries had a length of stay of up to two days at the Nakuru provincial hospital while $13 \%$ of them left the health facility just a day after admission [48]. The Kenya National Commission on Human Rights noted that some mothers were being discharged earlier than recommended due to overcrowding in maternity wards due to limited beds in view of the high demand for free delivery 
TABLE 1: Social demographics.

\begin{tabular}{|c|c|c|c|c|c|}
\hline \multicolumn{6}{|c|}{ Age } \\
\hline Mean & \multicolumn{2}{|c|}{ Standard deviation } & Maximum & \multicolumn{2}{|c|}{ Minimum } \\
\hline 26.0 & \multicolumn{2}{|c|}{6.2} & 49.0 & \multicolumn{2}{|c|}{13.0} \\
\hline \multicolumn{6}{|c|}{ Marital status } \\
\hline Single & \multicolumn{2}{|c|}{ Married } & Widowed & \multicolumn{2}{|c|}{ Divorced } \\
\hline $24.1 \%$ & \multicolumn{2}{|c|}{$72.7 \%$} & $1.2 \%$ & \multicolumn{2}{|c|}{$2.0 \%$} \\
\hline \multicolumn{6}{|c|}{ Religion } \\
\hline Christian & Islam & Hindu & $\begin{array}{c}\text { Traditional } \\
\text { Believes }\end{array}$ & \multicolumn{2}{|c|}{ Other } \\
\hline $89.0 \%$ & $9.7 \%$ & $0.5 \%$ & $0.4 \%$ & & \\
\hline \multicolumn{6}{|c|}{ Education level } \\
\hline Tertiary level & $\begin{array}{l}\text { Secondary } \\
\text { school } \\
\text { (completed) }\end{array}$ & $\begin{array}{c}\text { Secondary } \\
\text { school } \\
\text { (incomplete) }\end{array}$ & $\begin{array}{l}\text { Primary school } \\
\text { (completed) }\end{array}$ & $\begin{array}{l}\text { Primary school } \\
\text { (incomplete) }\end{array}$ & $\begin{array}{c}\text { Never been to } \\
\text { school }\end{array}$ \\
\hline $27.2 \%$ & $24.3 \%$ & $17.8 \%$ & $14.0 \%$ & $13.4 \%$ & $3.2 \%$ \\
\hline
\end{tabular}

TABLE 2: Satisfaction with services in the health facilities.

\begin{tabular}{lccc}
\hline Satisfaction Element & Not Satisfied & Neutral Response & Satisfied \\
\hline Consultation Time & $9.7 \%(215)$ & $35.6 \%(791)$ & $54.7 \%(1210)$ \\
Communication by the health care workers & $8.9 \%(173)$ & $29.7 \%(658)$ & $61.4 \%(1360)$ \\
Availability of staff in the delivery rooms & $12.6 \%(281)$ & $27.1 \%(600)$ & $60.3 \%(1335)$ \\
Availability of Staff in the wards & $14.9 \%(328)$ & $23.2 \%(515)$ & $61.9 \%(1373)$ \\
Cleanliness in the health facilities & $19.0 \%(421)$ & $40.7 \%(903)$ & $40.5 \%(892)$ \\
Privacy in the wards & $23.3 \%(515)$ & $37.1 \%(825)$ & $39.6 \%(856)$ \\
Availability of drugs and supplies & $18.5 \%(410)$ & $18.1 \%(402)$ & $63.4 \%(1404)$ \\
Mean for the total & $15.3 \%$ & $30.2 \%$ & $54.5 \%$ \\
\hline
\end{tabular}

services in Kenyan public health facilities [49]. Whereas shorter durations of stay in health facilities are recommended as part of service efficiency [50], mothers utilizing the free maternal healthcare services stayed in health facilities for an average of 3 days which is in line with the recommended postpartum hospital stay of 48 hours for uncomplicated vaginal delivery [51].

The study shows that beneficiaries of the free maternal healthcare policy are satisfied with communication by the healthcare workers, staff availability in the delivery rooms, availability of staff in the wards, and drug and supplies availability but unsatisfied with consultation time, cleanliness, and privacy in the wards. Low consultation time and poor hygienic environment may be attributed to a high demand for free delivery services against the few available healthcare workers while low privacy in health facilities could have been a result of sharing beds and incubators reported by the survey respondents [27]. A best fit linear regression model for the regressed variables is satisfaction $=12.674-0.108$ (days spent in the health facilities) +0.260 (number of ANC visits) +0.850 (sharing of incubators by newborns) +0.276 (effectiveness of pain control remedies) +0.399 (quality of meals provided) +0.845 (waiting time before being attended to) -1.44 (education level) + Error.

A 54.5\% satisfaction rate is in line with a previous study on satisfaction with delivery services which established that
$44 \%$ of the respondents were satisfied with the delivery services; and of those dissatisfied with the services, $12.1 \%$ of the respondents were dissatisfied with the cost implications [42]. This is an indication that cost is a minor factor in predicting satisfaction with delivery services and that there are other factors which account for contributing to satisfaction of users and they have not yet been addressed by the policy. The findings are also in line with a study at the Kakamega provincial hospital in western Kenya that shows that beneficiaries of the free maternal healthcare policy in the hospital were not satisfied with staff attitude (negligence and use of vulgar language), health facility environment, and privacy [52]. Abolishment of user fees in middle and low-income countries increases service utilization and consequently results in low quality of the services offered [5355]. Low consultation time may be attributed to demotivation of healthcare workers which has been observed in Uganda and Niger following the abolishment of user fees for delivery services [55-57].

There were no differences in satisfaction levels in rural and urban based facilities; however, satisfaction levels were highest in level 6 health facility (65.1\%) which is well staffed and equipped [58]. Through other studies, high education levels were negatively associated with satisfaction, a finding that has been reported by beneficiaries of free maternal healthcare services in Kakamega provincial hospital which 
TABLE 3: Analysis of satisfaction against major themes.

\begin{tabular}{|c|c|c|c|c|}
\hline Satisfaction Element & $\begin{array}{c}\text { Rural based } \\
\text { health } \\
\text { facilities }\end{array}$ & $\begin{array}{c}\text { Urban based } \\
\text { health } \\
\text { facilities }\end{array}$ & $\begin{array}{l}\text { Tier } 3 \text { health } \\
\text { facilities }\end{array}$ & $\begin{array}{c}\text { Tier } 4 \text { health } \\
\text { facility }\end{array}$ \\
\hline $\begin{array}{l}\text { Availability of Staff in } \\
\text { the delivery room }\end{array}$ & $61.7 \%$ & $59.2 \%$ & $59.0 \%$ & $84.6 \%$ \\
\hline $\begin{array}{l}\text { Availability of staff in the } \\
\text { ward }\end{array}$ & $61.5 \%$ & $62.3 \%$ & $61.2 \%$ & $80.3 \%$ \\
\hline $\begin{array}{l}\text { Availability of drugs and } \\
\text { supplies }\end{array}$ & $62.3 \%$ & $64.1 \%$ & $67.7 \%$ & $78.7 \%$ \\
\hline Privacy & $39.1 \%$ & $39.9 \%$ & $30.8 \%$ & $47.6 \%$ \\
\hline Cleanliness & $39.2 \%$ & $41 \%$ & $36.5 \%$ & $45.6 \%$ \\
\hline Communication & $62.1 \%$ & $60.8 \%$ & $64.5 \%$ & $62.8 \%$ \\
\hline Consultation time & $54.2 \%$ & $54.9 \%$ & $60.3 \%$ & $56.2 \%$ \\
\hline Overall Satisfaction & $54.30 \%$ & $54.60 \%$ & $54.3 \%$ & $65.11 \%$ \\
\hline
\end{tabular}

TABLE 4: Regression output.

\begin{tabular}{|c|c|c|c|c|c|c|c|}
\hline \multirow{2}{*}{ Model } & \multicolumn{2}{|c|}{ Unstandardized Coefficients } & \multirow{2}{*}{ Standardized Coefficients } & \multirow{2}{*}{$t$} & \multirow{2}{*}{ Sig. } & \multicolumn{2}{|c|}{$95 \%$ Confidence Interval for $B$} \\
\hline & $B$ & Beta & & & & Lower Bound & Upper Bound \\
\hline Constant & 12.674 & 0.859 & & 14.755 & 0.000 & 10.989 & 14.359 \\
\hline Days spent at the hospital & $1-0.108$ & 0.031 & -0.087 & -3.451 & 0.001 & -0.170 & -0.047 \\
\hline Number of ANC visits & 0.260 & 0.054 & 0.124 & 4.856 & 0.00 & 0.155 & 0.366 \\
\hline Incubator sharing & 0.850 & 0.250 & 0.087 & 3.405 & 0.001 & 0.360 & 1.340 \\
\hline Pain control remedies & 0.276 & 0.108 & 0.070 & 2.561 & 0.011 & 0.064 & 0.487 \\
\hline Meals & 0.399 & 0.137 & 0.088 & 2.914 & 0.004 & 0.130 & 0.668 \\
\hline Sanitation & 1.643 & 0.136 & 0.359 & 12.066 & 0.00 & 1.376 & 1.910 \\
\hline Warm shower water & 1.119 & 0.223 & 0.133 & 5.023 & 0.00 & 0.682 & 1.556 \\
\hline Time before attendance & 0.845 & 0.101 & 0.222 & 8.375 & 0.00 & 0.647 & 1.043 \\
\hline Education level & -0.144 & 0.070 & -0.053 & -2.067 & 0.039 & -.281 & -.007 \\
\hline
\end{tabular}

was included in this study [52]. Waiting time appears to be a key determinant of satisfaction with delivery services, a finding which is consistent with previous studies in Pumwani maternity home, Nyeri and Thika regions of Kenya, two of which were included in the study $[38,40]$. Similarly, effectiveness of pain control remedies and provision of adequate information in the health facilities are important in ensuring satisfaction with delivery services just as documented in Ethiopia and Botswana $[59,60]$.

Other experiences reported by beneficiaries of the free maternal healthcare policy include provision of a mosquito net $(49.3 \%)$, provision of warm water for showering $(52.8 \%)$, sharing of beds by mothers (43\%), and sharing of incubators by newborns $(26.8 \%)$ which point at low infrastructure and resource availability in the health facilities implementing the policy, a finding that has been reported by beneficiaries of the free maternal healthcare services at Machakos Level 5 hospital [61]. Following the abolishment of delivery fees in Kenya, beneficiaries reported a $13 \%$ dissatisfaction with the availability of beds at the Nakuru provincial hospital [48]. The Kenya National Commission on Human Rights (2013) noted that, due to the increased number of mothers delivering in health facilities following the implementation of the free maternal healthcare policy, maternity wards were overcrowded and some beneficiaries were forced to leave the hospital early to make room for others or even sleep on the floor due to lack of beds [62]. The counties highlighted by previous studies (Machakos and Nakuru) were not included in this particular study.

\section{Limitations}

Client satisfaction surveys are influenced by cultural response bias which is created by social and cultural factors that influence the way people perceive and respond to survey questions [63]. As such the satisfaction levels reported by the beneficiaries of the free maternal healthcare policy are based not only on their experiences in health facilities but also on social cultural factors. To ensure completeness in the responses, the beneficiary exit interviews questionnaires were interviewer administered. We cannot rule out a possible influence of this approach on responses given by the participants. Other limitations include the lack of a policy guideline outlining the full scope of services that should be offered under the free maternal healthcare policy; absence of variables in the questionnaire to capture place of previous delivery that would have aided in the analysis of satisfaction levels pre- and postpolicy implementation; and 
lack of absence of variables on ethnic and tribal background of respondents which may also influence satisfaction.

\section{Conclusion}

Mothers benefiting from the free delivery services are satisfied with communication by the healthcare workers, staff availability in the delivery rooms, availability of staff in the wards, and drug and supplies availability but unsatisfied with consultation time, cleanliness, and privacy in the wards. For continued use and recommendation of free delivery services to peers in Kenya, there is need to have changes in these institutional oriented independent predictors of satisfaction by the county governments and the national government.

\section{List of Abbreviations}

$\begin{array}{ll}\text { ADDRF: } & \text { African Doctoral Dissertation Research } \\ & \text { Fellowship } \\ \text { ANC: } & \text { Antenatal Clinic } \\ \text { BSc: } & \text { Bachelor of Science } \\ \text { BDS: } & \text { Bachelor of Dental Surgery } \\ \text { IDRC: } & \text { International Development Research } \\ & \text { Centre } \\ \text { MBChB: } & \text { Bachelor of Medicine, Bachelor of Surgery } \\ \text { MD: } & \text { Doctor of Medicine } \\ \text { MDG: } & \text { Millennium development goal } \\ \text { MMED: } & \text { Master of Medicine } \\ \text { NIH: } & \text { National Institutes of Health } \\ \text { PhD: } & \text { Doctor of Philosophy } \\ \text { PMRCPath: } & \text { Postgraduate Course, Royal College of } \\ & \text { Pathologists } \\ \text { SPSS: } & \text { Statistical Package for Social Sciences } \\ \text { UNITID: } & \text { Institute of Tropical and Infectious } \\ & \text { Diseases } \\ \text { USD: } & \text { United States dollar } \\ \text { WHO: } & \text { World Health Organization. }\end{array}$

\section{Data Availability}

The complete dataset used in this study is in the custody of Dr. Gitobu Cosmas Mugambi and is available to researchers upon written request.

\section{Ethical Approval}

Ethical approval was obtained from Kenyatta National Hospital and the University of Nairobi ethical committees. Administrative permission to conduct the study was obtained from the Kenyan Ministry of Health.

\section{Consent}

Written consent to participate in the study was sought from the respondents. Written consent to participate in the study was sought from the respondents.

\section{Conflicts of Interest}

The authors declare that they have no conflicts of interest.

\section{Authors' Contributions}

Dr. C. M. Gitobu, a doctoral candidate, conceived and designed this study under the guidance of Professor P. B. Gichangi and Professor W. O. Mwanda.

\section{Acknowledgments}

This research was partially funded by an African Doctoral Dissertation Research Fellowship (ADDRF) award offered by the African Population and Health Research Centre (APHRC) in partnership with the International Development Research Centre (IDRC).

\section{References}

[1] WHO, United Nations Children's Fund, United Nations Population Fund, The World Bank and the United Nations Population Division, Trends in Maternal Mortality: 1990 to 2013 Estimates, WHO Library Cataloguing-in-Publication Data, 2014.

[2] C. Oyston, C. F. Rueda-Clausen, and P. N. Baker, "Current challenges in pregnancy-related mortality," Obstetrics, Gynaecology and Reproductive Medicine, vol. 24, no. 6, pp. 162-169, 2014.

[3] L. Say, D. Chou, A. Gemmill et al., "Global causes of maternal death: a WHO systematic analysis," The Lancet Global Health, vol. 2, no. 6, pp. e323-e333, 2014.

[4] WHO, The Global Strategy for Women's, Children's and Adolescents' Health, 2016-2030, 2016, http://www.who.int/life-course/ partners/global-strategy/global-strategy-2016-2030/en/.

[5] Kenya National Bureau of Statistics, Kenya Demographic and Health Survey Report, 2013-2014, Ministry of Health, Kenya, 2015, http://medicalboard.co.k/resources/Kenya_Demographic_ and_Health_Survey_2014_Key_Indicators.pdf.

[6] O. I. Igwebueze, S. N. Obi, and E. O. V. Ugwu, "Impact of Free Maternal and Child Healthcare programme on maternal and neonatal healthcare outcome in Enugu State of Nigeria," Nigerian Journal of Medicine, vol. 20, no. 4, pp. 441-443, 2011.

[7] E. Arthur, "Wealth and antenatal care use: Implications for maternal health care utilisation in Ghana," Health Economics Review (HER), vol. 2, no. 1, pp. 1-8, 2012.

[8] D. H. Collins, J. D. Quick, S. N. Musau, and D. L. Kraushaar, "Health Financing Reform in Kenya: The Rise and Fall of Cost Sharing, 1989-1994," in Stubbs Monograph Series, vol. 1, Management Sciences for Health, Boston, 1996.

[9] Kenya National Bureau of Statistics, Kenya Demographic and Health Survey Report, 2008-2009, Ministry of Health, Kenya, 2010, http://apps.who.int/medicinedocs/documents/ s17116e/s17116e.pdf.

[10] J. N. Orem, F. Mugisha, C. Kirunga, J. MacQ, and B. Criel, "Abolition of user fees: The Uganda paradox," Health Policy and Planning, vol. 26, no. 2, pp. ii41-ii51, 2011.

[11] S. Bennett and L. Gilson, Health financing, Designing and implementing pro poor policies, DFID Health Systems Center, London, 2001.

[12] D. H. Collins, J. D. Quick, S. N. Musau, and D. L. Kraushaar, "Health Financing Reform in Kenya: The Rise and Fall of Cost Sharing," in Stubbs Monograph Series No.1, Management Sciences for Health, Boston, 1996.

[13] Kenya National Bureau of Statistics and ICF Macro, Kenya Demographic and Health Survey Report, 2008-2009, Ministry of Health, Kenya, 2010. 
[14] Ministry of Health, Kenya Household Health Expenditure and Utilization Survey, Nairobi: Ministry of Health, Kenya, 2003.

[15] G. Odwe, A. Agwanda, and A. Khasakhala, Barriers to Utilization of Health Facilities for Delivery in Kenya, 2015, http:// uaps2015.princeton.edu/uploads/151303\&gt.

[16] L. P. O. Were, E. Were, R. Wamai, J. Hogan, and O. Galarraga, "The Association of Health Insurance with institutional delivery and access to skilled birth attendants: Evidence from the Kenya Demographic and health survey 2008-09," BMC Health Services Research, vol. 17, no. 1, article 454, 2017.

[17] W.H.O., Universal health coverage; At least half the world lacks access to essential health services, 2017, http://www.who.int/ universal_health_coverage/en/\&gt.

[18] M. E. Kruk, "Universal health coverage: a policy whose time has come," BMJ (Clinical research ed.), vol. 347, p. f6360, 2013.

[19] W.H.O., Universal health coverage (UHC), 2017, http://www.who .int/mediacentre/factsheets/fs395/en/\&gt.

[20] J. Kitui, S. Lewis, and G. Davey, "Factors influencing place of delivery for women in Kenya: an analysis of the Kenya demographic and health survey, 2008/2009," BMC Pregnancy and Childbirth, vol. 13, article 40, 2013.

[21] Ministry of Health, The 2013 Kenya household health expenditure and utilization survey, 2013, https://www.healthpolicyproject.com/pubs/745_KHHUESReportJanuary.pdf.

[22] Ministry of Health, The Kenya National Oral Health Survey, 2015, https://profiles.uonbi.ac.ke/gathece/files/kenya_national_ oral_health_survey_report_2015.pdf\&gt.

[23] World Health Organization, The world health report: health systems financing: the path to universal coverage, 2010, http:// apps.who.int/iris/bitstream/10665/44371/1/9789241564021_eng .pdf.

[24] World Health Organization, Everybody business: strengthening health systems to improve health outcomes: WHO's framework for action, 2007, http://www.who.int/healthsystems/strategy/everybodys_business.pdf.

[25] L. E. Hatt, M. Makinen, S. Madhavan, and C. M. Conlon, "Effects of user fee exemptions on the provision and use of maternal health services: A review of literature," Journal of Health, Population and Nutrition, vol. 31, no. 4, pp. S67-S80, 2013.

[26] Ministry of Health Kenya, "Speech by H.E. Hon. Uhuru Kenyatta, C.G.H., President and Commander-in-Chief of the Defence Forces of the Republic of Kenya During the Madaraka Day Celebrations" (Nyayo National Stadium, June 1, 2013). 2013.

[27] E. Lang'at and L. Mwanri, "Healthcare service providers' and facility administrators' perspectives of the free maternal healthcare services policy in Malindi District, Kenya: A qualitative study," Reproductive Health, vol. 12, no. 1, article no. 59, 2015.

[28] E. W. Wamalwa, "Implementation challenges of free maternity services policy in kenya: The health workers' perspective," Pan African Medical Journal, vol. 22, article no. 375, 2015.

[29] WHO, Global Health Observatory (GHO) data-World Health Statistics 2017: Monitoring health for the SDGs, 2017, http://www .who.int/gho/publications/world_health_statistics/2017/en/\&gt.

[30] R. Amdemichael and M. Tafa, "Maternal satisfaction with the delivery services in Assela Hospital, Arsi zone, Oromia region," Gynecology \& Obstetrics, vol. 4, article 257, 2014.

[31] R. Tesfaye, A. Worku, W. Godana, and B. Lindtjorn, "Client Satisfaction with Delivery Care Service and Associated Factors in the Public Health Facilities of Gamo Gofa Zone, Southwest Ethiopia: In a Resource Limited Setting," Obstetrics and Gynecology International, vol. 2016, Article ID 5798068, 2016.
[32] E. S. Bazant and M. A. Koenig, "Women's satisfaction with delivery care in Nairobi's informal settlements," International Journal for Quality in Health Care, vol. 21, no. 2, pp. 79-86, 2009.

[33] World Health Organization, Making Pregnancy Safer: The Critical Role of the Skilled Attendant: A Joint Statement by WHO, ICM, FIGO, World Health Organization (WHO), Geneva, Switzerland, 2004, http://www.who.int/maternal_child_adolescent/documents/9241591692/en/.

[34] H. H. M. Zaky, H. A. S. Khattab, and D. Galal, "Assessing the quality of reproductive health services in Egypt via exit interviews," Maternal and Child Health Journal, vol. 11, no. 3, pp. 301306, 2007.

[35] A. H. Al-Doghaither, B. M. Abdelrhman, and A. A. Wahid Saeed, "Patients' satisfaction with physicians' services in primary healthcare centres in Kuwait City, Kuwait," Journal of The Royal Society for the Promotion of Health, vol. 120, no. 3, pp. 170$174,2000$.

[36] D. Debono and J. Travaglia, "Complaints and Patient Satisfaction: A Comprehensive Review of the Literature," in The Centre for Clinical Governance Research in Health, University of New South Wales, Australia, 2009.

[37] A. Chow, E. K. Mayer, A. W. Darzi, and T. Athanasiou, "Patientreported outcome measures: the importance of patient satisfaction in surgery," Surgery, vol. 146, no. 3, pp. 435-443, 2009.

[38] M. W. Nyongesa, R. Onyango, and R. Kakai, "Determinants of clients' satisfaction with healthcare services at Pumwani Maternity Hospital in Nairobi - Kenya," International Journal of Social and Behavioural Sciences, vol. 2, no. 1, pp. 011-017, 2014.

[39] N. K. Wanjau, B. W. Muiruri, and E. Ayodo, "Factors Affecting Provision of Service Quality in the Public Health Sector: A Case of Kenyatta National Hospital," International Journal of Humanities and Social Science, vol. 2, no. 13, 2012.

[40] J. M. Wambua, R. Mbayaki, P. M. Munyao et al., "Client satisfaction determinants in four Kenyan slums," International Journal of Health Care Quality Assurance, vol. 28, no. 7, pp. 667-677, 2015.

[41] National Coordinating Agency for Population and Development, Maternal Deaths on the Rise in Kenya: A Call to Save Women's Lives, policy brief No. 9, A publication of the National Coordinating Agency for Population \& Development, 2010, https://www.google.com/url.

[42] E. Wubs, D. Brals, M. Van der List, and C. Elbers, Lessons learned from a maternal health study in Nandi County, Kenya. Learning \& analysis brief, Amsterdam Institute for Global Health and Development, Pharm Access Foundation, Healthcare quality, 2016.

[43] Reference: Government of Kenya, Module 1 the Healthcare System In Kenya Unit 1.1: The Healthcare System in Kenya, 2017.

[44] D. K. Kombo and D. L. Tromp, "Proposal and thesis writing: An introduction," in Nairobi: Pauline Publications Africa, 2006, http://jaysean.com/index.php.

[45] Ministry of Health Kenya, Kenya master health facility list, 2015, http://kmhfl.health.go.ke/\#/home\&gt.

[46] W. Bill-Godden, Sample size determination for finite populations, 2004, http://www.williamgodden.com.

[47] National Institutes of Health (NIH), NIH Research Ethics Training, 2015, https://researchethics.od.nih.gov/\&gt.

[48] J. M. Murima, The effect of free maternal health care services on perceived quality of service delivery at Nakuru Provincial General Hospital, Strathmore University, 2016, http://su-plus.strathmore.edu/handle/11071/4583. 
[49] Kenya National Commission on Human Rights, Implementing Free Maternal Health Care in Kenya: Challenges, Strategies, and Recommendations, 2013, http://www.knchr.org/Portals/0/EcosocReports/Implementing\%20Free\%20Maternal\%20Health\% 20Care\%20in\%20Kenya.pdf\&gt.

[50] J. Bowers and H. Cheyne, "Reducing the length of postnatal hospital stay: Implications for cost and quality of care," $B M C$ Health Services Research, vol. 16, no. 1, article no. 1214, 2016.

[51] A. P. Eaton, "Early Postpartum Discharge: Recommendations From a Preliminary Report to Congress," Pediatrics, vol. 107, no. 2, pp. 400-403, 2001.

[52] B. Mukabana, A. Kwena, and T. Wambui, "Effects of the free maternity care program on utilization of services at a county referral hospital in Kenya," Kenyan Journal of Nursing \& Midwifery, vol. 1:2, pp. 132-144, 2016.

[53] M. Lagarde and N. Palmer, "The impact of user fees on health service utilization in low- and middle-income countries: how strong is the evidence?" Bulletin of the World Health Organization, 2016, http://www.who.int/bulletin/volumes/86/11/07049197/en/.

[54] V. Ridde and F. Morestin, "A scoping review of the literature on the abolition of user fees in health care services in Africa," Health Policy and Planning, vol. 26, no. 1, pp. 1-11, 2011.

[55] G. M. Burnham, G. Pariyo, E. Galiwango, and F. WabwireMangen, "Discontinuation of cost sharing in Uganda," Bulletin of the World Health Organization, vol. 82, no. 3, pp. 187-195, 2004.

[56] J. Nabyonga Orem, F. Mugisha, C. Kirunga, J. MacQ, and B. Criel, "Abolition of user fees: The Uganda paradox," Health Policy and Planning, vol. 26, no. 2, pp. ii41-ii51, 2011.

[57] V. Ridde and A. Diarra, "A process evaluation of user fees abolition for pregnant women and children under five years in two districts in Niger (West Africa)," BMC Health Services Research, vol. 9, article no. 89, 2009.

[58] Ministry of Health Kenya and WHO, Kenya Service Availability and Readiness Assessment Mapping, A Comprehensive mapping of health services, capacity for service provision, sector investments and readiness to provide services by County (SARAM) REPORT, 2013.

[59] A. R. Bamidele, M. E. Hoque, and H. van der Heever, "Patient satisfaction with the quality of care in a primary health care setting in Botswana," South African Family Practice, vol. 53, no. 2, pp. 170-175, 2011.

[60] T. Melese, Y. Gebrehiwot, D. Bisetegne, and D. Habte, "Assessment of client satisfaction in labor and delivery services at a maternity referral hospital in Ethiopia," Pan African Medical Journal, vol. 17, article 76, 2014.

[61] O. D. Orare, A. Wangombe, F. Muchiri, M. Chimbevo, and W. Ooga, "The Roles of Infrastructure and Resources on Implementation of Free Maternal Healthcare Services in Machakos Level 5 Hospital, Machakos County, Kenya," Science Journal of Public Health, vol. 5, no. 1, pp. 49-55, 2017.

[62] Kenya National Commission on Human Rights, "Implementing Free Maternal Health Care in Kenya: Challenges, Strategies, and Recommendations, 2013," http://www.knchr.org/Portals/0/ EcosocReports/Implementing\%20Free\%20Maternal\%20Health\% 20Care\%20in\%20Kenya.pdf\&gt.

[63] A. Gayet-Ageron, T. Agoritsas, L. Schiesari, V. Kolly, and T. V. Perneger, "Barriers to participation in a patient satisfaction survey: Who are we missing?” PLoS ONE, vol. 6, no. 10, Article ID e26852, 2011. 


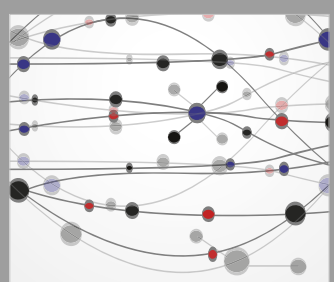

The Scientific World Journal
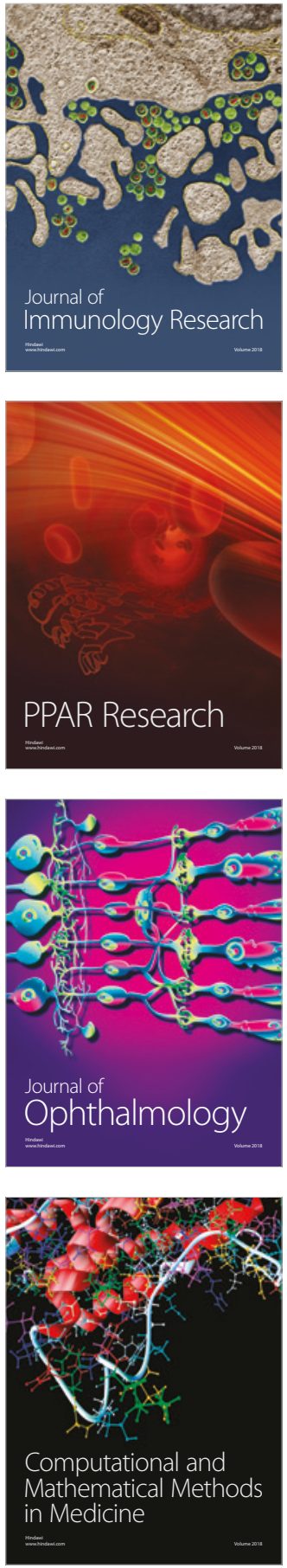

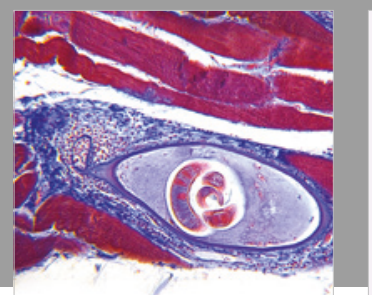

Gastroenterology Research and Practice

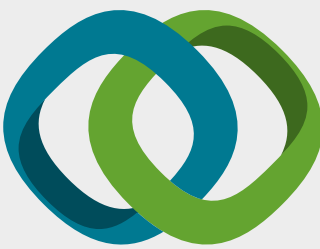

\section{Hindawi}

Submit your manuscripts at

www.hindawi.com
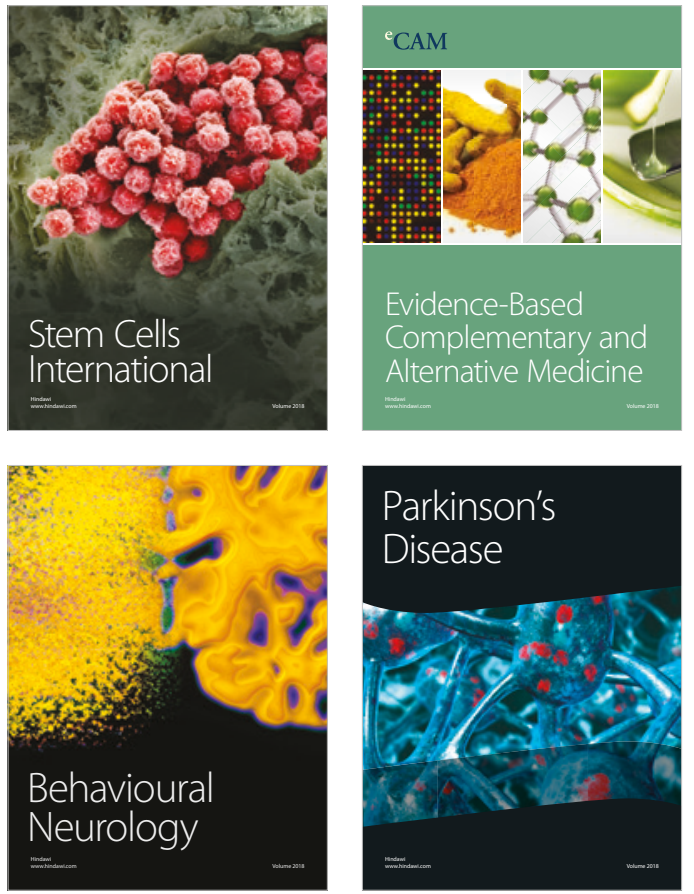

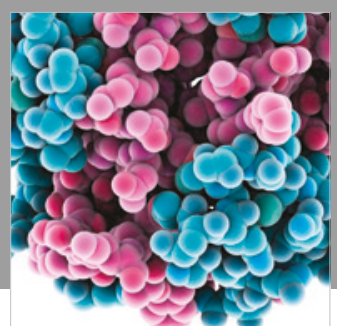

ournal of

Diabetes Research

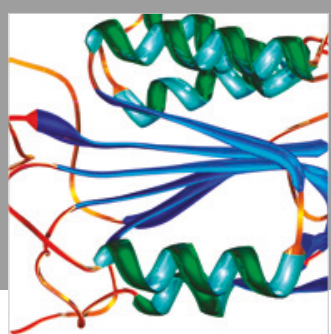

Disease Markers
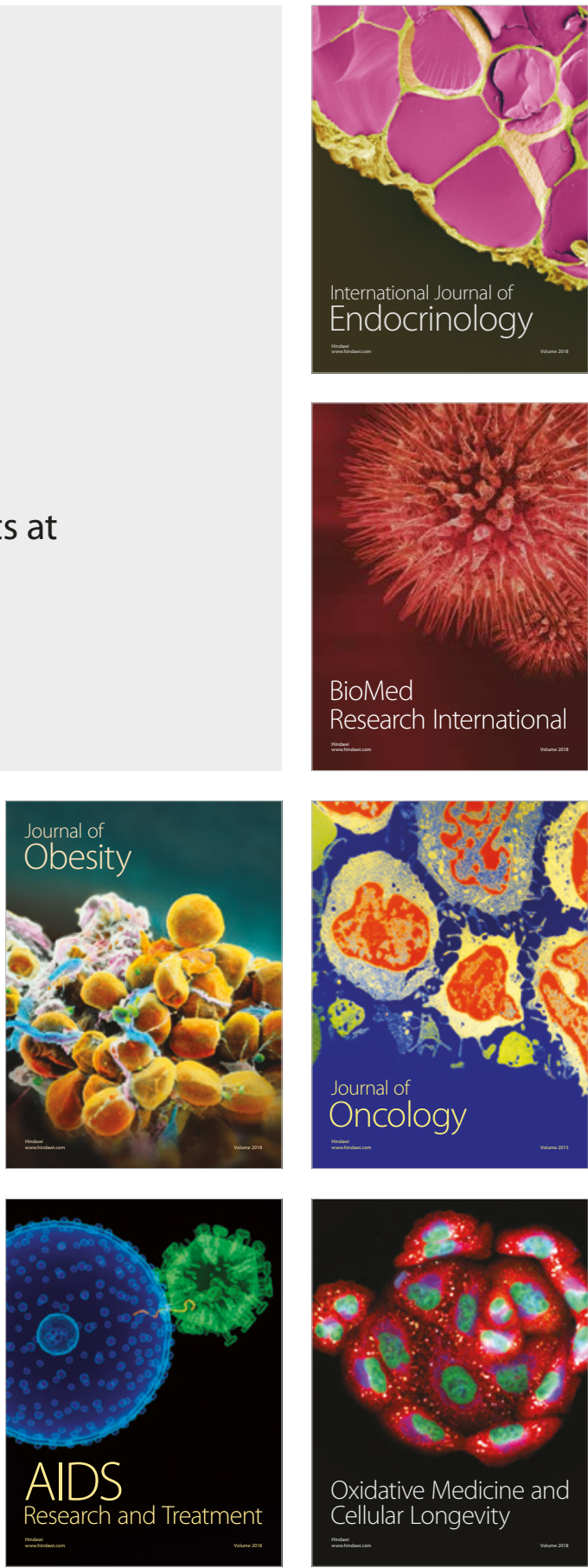